\begin{tabular}{c|c|c}
\hline \hline $\begin{array}{c}\text { Vol. 377: 213-225, 2009 } \\
\text { doi: } 10.3354 / \mathrm{meps} 07825\end{array}$ & $\begin{array}{c}\text { MARINE ECOLOGY PROGRESS SERIES } \\
\text { Mar Ecol Prog Ser }\end{array}$ & Published February 26 \\
\hline \hline
\end{tabular}

\title{
Fish life histories and marine protected areas: an odd couple?
}

\author{
David M. Kaplan* \\ Institut de Recherche pour le Développement (IRD), Centre de Recherche Halieutique Méditerranéenne et Tropicale, \\ av. Jean Monnet, BP 171, 34203 Sète cedex, France
}

\begin{abstract}
Though marine protected areas (MPAs) have recently become a central element of many marine resource management plans, the potentially negative effects of MPAs for fisheries harvests remain the major impediment to their use. Understanding population responses to implementation of MPAs, and their consequences for harvests, is essential to assessing the value of MPAs as a management tool. Here I use a simple model to link what we know best about marine species, namely how fast they grow and how much they reproduce, with what we want to know, namely what will be the effects of spatial management efforts. Specifically, I show that whether maximum sustainable yield (MSY) or yield at fixed total recruitment increases with MPAs can be determined by an intuitive equation comparing the change in average individual biomass (i.e. harvestable material) with the change in individual lifetime reproduction due to increasing reserve area. Application of this model to different population structures demonstrates that initial MPA results indicating MSY would be unchanged with reserves for species with simple life-histories do not hold up for species for which biomass, reproduction or mortality vary with age. This explains many negative MPA results from age-structured models of optimally exploited species. On the other hand, the model predicts that species harvested later in life, and which begin reproduction after first harvest, may benefit from MPAs. Nevertheless, for these increases to occur, the age at first reproduction must in general be a considerable time after the age at first harvest.
\end{abstract}

KEY WORDS: Marine protected area - MPA · Life history traits · Growth rates · Fisheries management $\cdot$ Density dependence $\cdot$ Harvesting strategy $\cdot$ Maximum sustainable yield $\cdot$ MSY

Resale or republication not permitted without written consent of the publisher

\section{INTRODUCTION}

Marine protected areas (MPAs, also referred to here as marine reserves) are becoming an essential element of marine resource management. They have attracted wide scientific interest due to their potential to improve the conservation status and fisheries yield of marine resources, as well as their utility for studying marine population and ecosystem dynamics (see e.g. Gerber et al. 2003, Sale et al. 2005 and references therein). Despite this wide interest, implementation of MPAs remains a difficult task, in large part due to uncertainty about the impacts their implementation will have on surrounding fisheries. Ultimately, the principal goal of MPAs is to reduce anthropogenic pressures on marine ecosystems; therefore, under- standing their impacts on fisheries and vice-versa is central to achieving the conservation and fisheries goals of MPAs.

Numerous modeling studies have considered how larval and adult dispersal (Gerber et al. 2005, Kaplan 2006), recruitment processes (Gaylord et al. 2005, White \& Kendall 2007), and economic considerations (Sanchirico et al. 2006, White et al. 2008) affect fisheries harvests with MPA. These studies have established several key results regarding the effects of MPAs: (1) fishery yield increases with reserves relative to conventional management alone for severely overfished species (Hart 2006), (2) maximum sustainable yield (MSY) is equivalent with and without reserves in simple models (Hastings \& Botsford 1999), and (3) this equivalence breaks down when size-structure (Hart 2006), inter-co- 
hort density dependence (White \& Kendall 2007) and/or economics considerations (Smith \& Wilen 2003) are added to models. Though these results provide a global understanding of the expected consequences of MPA implementation for fisheries harvests, a general understanding of how basic species life-history traits, such as growth and reproductive rates, are likely to enhance or decrease the value of MPAs as a management tool is lacking. This general understanding is necessary to identify priority species for management with MPAs and to interpret the population response of marine species to MPA implementation.

It is well known that species with low reproductive rates and long life expectancy are often more susceptible to overfishing than species with faster generation times (Adams 1980, Jennings et al. 1998). Therefore, it is natural to expect that the same life-history traits (reproductive rates, growth rates, etc.) are important for the response of a species to MPA implementation. Here a simple model is used to assess how basic life-history traits impact harvests when MPAs are combined with effective conventional effort-based fisheries management. The model indicates that a simple and intuitive relationship comparing changes in biomass with changes in reproductive capacity governs whether yield will increase with MPAs. This model is applied to a series of populations with progressively more complex life histories, ending with a population with a non-linear agelength relationship, allometric relationships for biomass and reproductive capacity as a function of length, and non-zero ages of first harvest and first reproduction. This life-history structure is representative of a wide class of species and provides an overall view of what conditions are necessary for well-managed species to benefit from MPA implementation.

This study complements previous results in at least 2 important ways. First, the equivalence in yield identified by Hastings \& Botsford (1999) does not extend to more realistic MPA models; however, the biological mechanisms that break this equivalence have not been adequately identified. Exactly where this equivalence breaks down is determined here by connecting results from populations with different levels of complexity in their life histories. Second, while many studies have explored how MPAs might help in cases of failed conventional fisheries management, it is now widely recognized that both MPAs and effective conventional management are necessary. Most studies addressing the effects of MPAs in the presence of effective conventional fisheries management have found that harvests decrease with reserves (e.g. Kaplan 2006), although there are notable exceptions (Gaylord et al. 2005, White \& Kendall 2007). Here, these results are explained in a general context by relating life-history traits to yield with MPAs.

\section{METHODS}

Model formulation. The MPA system is described in terms of a 2 patch model (reserve, fished area) of a species with sedentary adults, well-mixed larvae, and post-settlement intra-cohort density-dependent recruitment. This structure approximately represents many of the species targeted for management with MPAs, namely those that are relatively sedentary as adults, but possess an extended planktonic larval phase suggestive of long-distance dispersal (although our understanding of true larval dispersal patterns is far from complete; see the 'Discussion' for thoughts on finite dispersal distances). For the sake of this manuscript, a MPA or marine reserve represents a zone where the species in question is not fished.

The model used here, originally formulated in Hart (2006), is described at equilibrium by 2 equations:

$$
\begin{gathered}
R=s(S)=s(b R)=s\left(\left(C b_{0}(0)+(1-C) b_{0}(F)\right) R\right) \\
Y=y R=(1-C) y_{0}(F) R
\end{gathered}
$$

where $R$ and $Y$ are the recruitment and yield, respectively, per unit of habitat area, $s(S)$ is the post-settlement, density-dependent recruitment function at settlement rate $S, F$ is the fishing mortality rate per unit area outside reserves, $C$ is the fraction of the habitat area in reserves, $y$ and $b$ are the average (over all habitat areas) of yield per recruit (YPR) and lifetime egg production per recruit (LEP), respectively, and $y_{0}(F)$ and $b_{0}(F)$ are the YPR and LEP, respectively, as a function of the local fishing rate. YPR and LEP describe the average harvestable biomass and number of young, respectively, that a typical individual produces over its lifetime.

The dynamics of the system are determined by the first line of Eq. (1), which describes how the reproductive activity of individual recruits contributes to the total settlement and, via the settler-recruit relationship, to the arrival of new recruits. Settlement, the term inside the outermost parentheses, is defined in terms of the total number of recruits, the fraction of total area in each patch and the average number of eggs produced by an individual recruit to each patch. The settlerrecruit relationship, $s(S)$, describes the set of densitydependent effects that determine the number of fully recruited individuals resulting from a certain number of settlers. As settlement and recruitment are not functions of space, it is implicitly assumed that larval dispersal sufficiently mixes larvae so that settlement is uniform over space and that the settler-recruit relationship is not location specific. The model is an equilibrium model, not unlike that used in Kaplan et al. (2006), because a stable age distribution is required for the number of eggs produced at a given moment to be 
accurately reflected by the product of the recruitment level and the LEP.

Hart (2006) showed using this model that yield increases with area in reserves at a fixed fishing rate (though total effort changes as the area open to fishing decreases) if the slope of the post-settlement density dependence at the settlement rate is greater than the inverse of the value of natural, unfished LEP. This condition is satisfied for all species at a high enough fishing rate and can be used to determine a fishing rate above which reserves will increase yield. As such, it is most likely to be satisfied by species that are heavily exploited, though the condition is globally applicable.

The same model can be used to address a somewhat different question: For fisheries that are 'effectively' managed using conventional methods, when can creating or expanding area in marine reserves increase fisheries yield? By effective conventional fisheries management, I refer to effort-based controls on fishing activity that are successful at limiting take and presumably assure persistence of the population. The question of MPA consequences for well-managed species is central to many MPA implementation efforts that wish to know if and how MPAs will complement existing conventional management efforts. Conventional, effort-based management goals often involve maintaining a certain level of total recruitment to the adult population that is considered sufficient to ensure persistence and produce reasonable fisheries yield. This can be achieved either directly by monitoring recruitment events (e.g. via regular juvenile salmon or rockfish surveys in California, USA) and using this information to adjust catch levels, or indirectly by maintaining a certain level of spawning-stock-biomass (SSB) (or, similarly, LEP) in the adult population that is presumed to produce sufficient new individuals for species persistence. From the first line of Eq. (1), one can maintain a constant overall recruitment rate, $R$, while changing the fraction of area in reserves if the fishing rate is simultaneously increased in non-reserve areas so as to maintain the spatial average LEP per recruit, $b$, constant. This scenario could occur either directly through adjustments to effort restrictions to account for protection via MPAs or indirectly due to the displacement of fishing effort from newly-created reserve areas to remaining fished areas in the absence of appropriate controls to limit such effort concentration. If one considers infinitesimal increases in the fraction of the total habitat area in reserves, $\Delta C$, and in the fishing rate, $\Delta F$, then $b$ is constant if:

$$
\Delta b=\left[b_{0}(0)-b_{0}(F)\right] \Delta C+(1-C) b_{0}^{\prime}(F) \Delta F=0
$$

where $b^{\prime}{ }_{0}(F)$ is the derivative of $b_{0}(F)$ with respect to $F$. This can be rearranged to define $\Delta F$ in terms of $\Delta C$ at fixed total recruitment:

$$
\begin{aligned}
\frac{\Delta C}{1-C} & =\frac{-b_{0}^{\prime}(F)}{b_{0}(0)-b_{0}(F)} \Delta F \\
& =\frac{\mathrm{d}\left[\ln \left(b_{0}(0)-b_{0}(F)\right)\right]}{\mathrm{d} F} \Delta F
\end{aligned}
$$

This equation essentially states that the fractional change in the habitat area open to fishing must be equal to the fractional increase in the difference between LEP inside and outside reserves to maintain total reproductive output constant.

Now we wish to determine when these changes in area in reserves and fishing rate increase yield. As the absolute value of yield is not of interest, fractional changes in yield will be examined:

$$
\frac{\Delta Y}{Y}=\frac{-\Delta C}{1-C}+\frac{y_{0}^{\prime}(F)}{y_{0}(F)} \Delta F>0
$$

Eliminating $\Delta C$ from Eq. (4) via substitution of Eq. (3) produces:

$$
\left[\frac{\mathrm{d}\left[\ln \left(y_{0}(F)\right)\right]}{\mathrm{d} F}-\frac{\mathrm{d}\left[\ln \left(b_{0}(0)-b_{0}(F)\right)\right]}{\mathrm{d} F}\right] \Delta F>0
$$

Therefore, fisheries yield will increase due to an infinitesimal increase in the fraction of area in reserves at fixed total recruitment if:

$$
\frac{\mathrm{d}\left[\ln \left(y_{0}(F)\right)\right]}{\mathrm{d} F}-\frac{\mathrm{d}\left[\ln \left(b_{0}(0)-b_{0}(F)\right)\right]}{\mathrm{d} F}>0
$$

This quantity gives the fractional increase in yield per unit increase in fishing rate (combined with an appropriate increase of the habitat area in reserves).

This final relationship has an intuitive interpretation. The sensitivity of YPR to changes in the fishing rate must be greater than the sensitivity of LEP to changes in the fishing rate in order for yield to increase with reserves. This simple condition guarantees that the decrease in area open to fishing will be overcompensated by increased catch in the remaining harvested areas due to the increase in fishing rate. This overcompensation can only occur if YPR increases faster than LEP decreases.

While MSY is no longer regularly used as the management goal, it is still a valuable and often-used reference point for setting relative target population levels and assessing fisheries performance (e.g. Mace 2001). The condition described above is sufficient to ensure that MSY will increase with increasing area in reserves (when the quantity in Eq. 6 is evaluated at the fishing rate that produces MSY) as at least one combination of increased reserve area and increased fishing rate produces greater yield. Using the results of Hart (2006), Appendix 1 shows that this condition is also necessary for MSY to increase. Therefore, the same condition applies to management for either a fixed level of total recruitment or for MSY. As such, this condition provides an estimate of what 'good' combined management (i.e. management that ap- 
proaches maximum yield) might produce in terms of fisheries benefits. Furthermore, the Appendix 1 shows that this condition for increased yield is equivalent to that developed in Hart (2006). Though valid only at MSY, it is in some ways easier to apply than that of Hart (2006) (see next paragraph).

Note that inter-cohort post-settlement density dependence does not enter into Eq. (6). As such, Eq. (6) can be evaluated for a wide variety of life histories without reference to the settler-recruit relationship (though calculating the fishing rate that produces MSY does require this). This is extremely advantageous in terms of our ability to evaluate Eq. (6), given that estimates of settler-recruit relationships in most marine species suffer from considerable uncertainties while basic life-history parameters related to growth and reproduction can often be estimated with relatively high precision. Furthermore, Eq. (6) is insensitive to certain details of the life history of a species. For example, as the quantity involves only sensitivities, multiplicative factors in $y_{0}(F)$ and $b_{0}(0)-b_{0}(F)$ that are independent of the fishing rate (e.g. multiplicative constants) do not affect the value of the inequality. Furthermore, additive terms in $b_{0}(F)$ that are independent of the fishing rate (e.g. reproductive activity of individuals before the age of first harvest) also do not contribute to Eq. (6). In summary, Eq. (6) is invariant up to redefinitions of YPR and LEP of the form:

$$
\begin{gathered}
\tilde{y}_{0}(F)=\alpha y_{0}(F) \\
\tilde{b}_{0}(F)=\beta b_{0}(F)+\gamma
\end{gathered}
$$

where $\tilde{b}_{0}(F)$ and $\tilde{y}_{0}(F)$ are equivalent (in terms of the value of Eq. 6), redefinitions of YPR and LEP, and $\alpha, \beta$ and $\gamma$ are not functions of F. When Eq. (6) is applied to different life histories below, these redefinitions will often be explicitly or implicitly invoked (for example, by ignoring constants of proportionality or not considering reproductive activity before the onset of fishing).

One immediate consequence of Eq. (6) is that, assuming $b_{0}(F)$ is a decreasing function of $F$, this condition can only be satisfied if $y_{0}(F)$ increases with $F$. Therefore, any species that is fished beyond its maximum value of YPR cannot increase total yield by increasing the area in reserves at fixed total recruitment. This does not necessarily mean that yield cannot be increased with reserves in these cases, but rather that yield cannot increase without increasing recruitment (Hart 2006 provides the diagnostic tools needed for these cases). Therefore, Eq. (6) is applicable only for effectively managed fisheries that have not pushed the population beyond maximum YPR. Furthermore, it is possible for the total derivative of yield with respect to area in reserves at fixed overall recruitment to be positive, but the partial derivative (i.e. the change in yield at fixed fishing rate) to be negative (which can be determined by the condition in Hart 2006). In these cases, fixing total recruitment will increase yield, but is not the optimal management strategy in the sense that there exist ways to decrease area in reserves while increasing fishing rate that will produce higher yields.

Catch per unit effort (CPUE). The same logic used above to look at total yield can also be used to examine catch per unit of fishing effort (CPUE) at fixed total recruitment. Assuming that fishing effort is accurately represented by fishing mortality, the total effort in this 2 patch system is simply $(1-C) F$. Therefore, the CPUE is given by:

$$
\text { CPUE }=y_{0}(F) \frac{R}{F}
$$

and the fractional change in CPUE at fixed total recruitment is:

$$
\frac{\Delta \mathrm{CPUE}}{\mathrm{CPUE}}=\left[\frac{\mathrm{d}\left[\ln \left(y_{0}(F)\right)\right]}{\mathrm{d} F}-\frac{1}{F}\right] \Delta F
$$

Therefore, the fractional change in CPUE is proportional to the following quantity:

$$
\frac{\mathrm{d}\left[\ln \left(y_{0}(F)\right)\right]}{\mathrm{d} F}-\frac{1}{F}
$$

This quantity will be greater than 0 if the slope of $y_{0}(F)$ is greater than that of a line from the origin to the point $\left\{F_{1} y_{0}(F)\right\}$, as can be seen by multiplying the entire expression by $y_{0}(F)$ and taking the derivative of the logarithm. This condition is likely to be difficult to satisfy, as the rate of increase in $y_{0}(F)$ generally decreases with increasing $F$ due to the increasingly small size of the fish caught; therefore, $y_{0}(F)$ tends to be globally convex, which is incompatible with this condition.

\section{RESULTS}

While Eqs. (6) and (10) provide intuitive relationships for determining how life-history characteristics affect total yield and CPUE, a more detailed understanding can only be gained by application of these relationships to a variety of different life histories. Here a series of populations with progressively more complex life-history characteristics is examined, beginning with simple discrete-time populations for whom yield is measured in numbers of fish instead of biomass and ending with a complex age-structured population with von Bertalanffy (non-linear) growth and age limits for first reproduction and entry into the fishery. The range of populations presented is of interest because it represents a wide variety of species and MPA model populations, and in general demonstrates the conditions under which reserves will tend to have higher yield than effort-based management alone. 


\section{A simple discrete population with maximum age}

Hastings \& Botsford (1999) demonstrated for a simple iteroparous population with sedentary adults, well-mixed larvae and post-settlement density dependence that MSY is equivalent with and without reserves. A somewhat more general version of this same model is examined here so as to demonstrate the continuity between the approach used here and that of Hastings \& Botsford (1999). Consider a population for whom natural mortality, fishing mortality and reproduction are discrete, independent processes, as might be appropriate for a species with a very limited reproductive period that occurs just after a short fishing season. I assume that each process occurs at every time step in the order mentioned, that the number of eggs produced and the harvestable biomass are directly proportional to the number of individuals and independent of age, and that individuals live up to a fixed maximum age $k$. That yield is proportional to the number of individuals caught and not to the biomass is a particularly important and unrealistic assumption. This assumption assures that YPR will increase indefinitely with fishing rate, which will tend to favor increases in CPUE (or similarly total yield) with reserves, as noted following Eq. (10). Though unrealistic, results for this population structure provide an interesting contrast to those from more realistic populations examined below for whom yield is measured in biomass.

Because one is interested in per recruit quantities, only the probability of survival and capture by the fishery for an individual recruit need be examined. The probability of survival of an individual recruit to age $i$ is given by:

$$
n_{i}=(1-h)^{i} a^{i}
$$

where $a$ is the adult survival rate and $h$ is the harvest fraction per unit time. Note that without loss of generality, the fishing rate, $F$, can be replaced by harvest fraction, $h$, in Eq. (6).

The YPR and LEP, respectively, for this species are:

$y_{0}(h)=h a \sum_{i=1}^{k} n_{i-1}=h a \sum_{0}^{k-1}(1-h)^{i} a^{i}=h a \frac{1-(1-h)^{k} a^{k}}{1-(1-h) a}$

and

$$
\begin{gathered}
b_{0}(h)=\sum_{1}^{k} n_{i}=\sum_{1}^{k}(1-h)^{i} a^{i}=\sum_{0}^{k}(1-h)^{i} a^{i}-1 \\
b_{0}(h)=\frac{1-(1-h)^{k+1} a^{k+1}}{1-(1-h) a}-1 \\
b_{0}(h) \approx \frac{1-(1-h)^{k+1} a^{k+1}}{1-(1-h) a}
\end{gathered}
$$

Note that constants of proportionality have been ignored and, in the final expression for LEP, the additive constant has been dropped, as these are not important for Eq. (6) (see Eq. 7 and preceding discussion).
Though an analytic expression for Eq. (6) can be found for this population, it is complex and not directly informative in the absence of numerical evaluation. There are, however, 2 interesting limits where the expression simplifies. The first is that of an infinite maximum age. This case replicates the model in Hastings \& Botsford (1999). Substitution of Eqs. (12) and (13) into Eq. (6) for $k=\infty$ reveals that the 2 sensitivities are identical and, therefore, yield at fixed total recruitment or MSY is unchanged with increasing area in reserves, as shown by Hastings \& Botsford (1999).

The other limit where Eq. (6) simplifies is that of an obligate semelparous population (i.e. $k=1$ ). In this case, the 2 sensitivities again cancel and yield is unchanged with reserves.

Given these 2 results, it is tempting to assume that the 2 sensitivities cancel for all values of $k$. However, this is not the case. For all other values of $k$, numerical evaluation of Eq. (6) reveals that it is negative for all values of $a$ and $h$; therefore, yield decreases with reserves. Decreases are greatest for populations with average longevities approaching the maximum age (i.e. a close to 1), but approaches 0 for species with high longevities or large $k$ (Fig. 1).

\section{Discrete reproduction and continuous mortality}

Next, a population for whom natural and fishing mortalities are continuous processes but reproduction is a discrete-time process is examined. This structure could represent an iteroparous species with synchronous reproduction and a year-round fishery. It also serves as a bridge between the discrete population

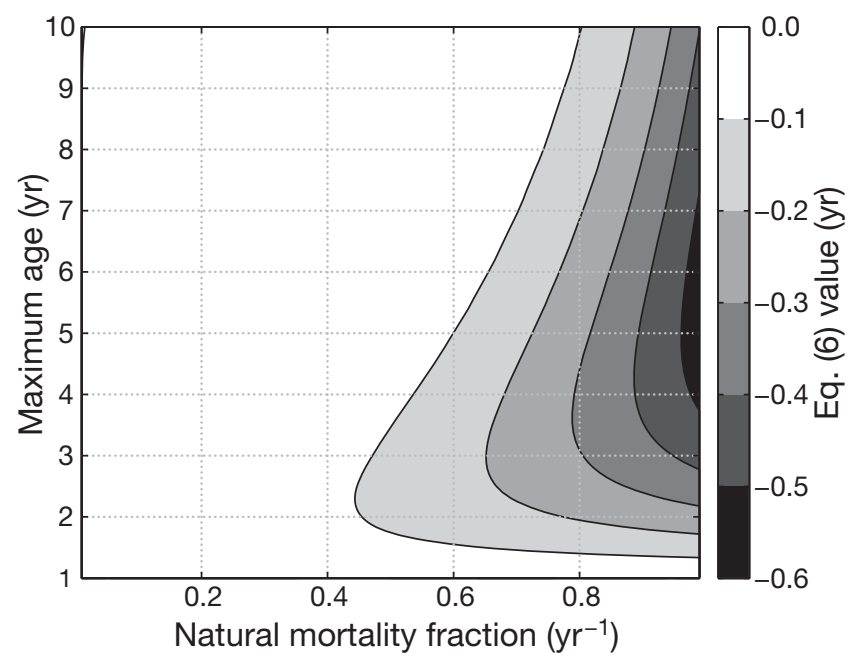

Fig. 1. Value of Eq. (6) for a population with discrete mortality and reproduction as a function of natural mortality fraction and maximum age. Harvest fraction is fixed at 0.5 
considered above and the continuous populations examined in the following sections.

It is assumed that individuals can live up to a fixed maximum age and are subject to fishing and natural mortality over their entire adult lifetimes. Individuals reproduce once per time step, and biomass and reproductive capacity are directly proportional to the number of individuals. The probability of survival of an individual recruit to age $a$ is given by:

$$
n(a)=\mathrm{e}^{-(M+F) a}
$$

where $M$ is the natural mortality rate. Given this population structure, and that biomass and reproductive capacity are strictly proportional to the number of individuals, the YPR and LEP per recruit are given by:

$$
\begin{gathered}
y_{0}(F)=\sum_{1}^{k}[n(a-1)-n(a)] \frac{F}{M+F}=\frac{F}{M+F}[n(0)-n(k)]= \\
\frac{F}{M+F}\left[1-\mathrm{e}^{-(M+F) k}\right]
\end{gathered}
$$

and

$$
\begin{gathered}
b_{0}(F)=\sum_{1}^{k} n(a)=\sum_{1}^{k} \mathrm{e}^{-(M+F) a}=-1+\sum_{0}^{k} \mathrm{e}^{-(M+F) a}= \\
\frac{1-\mathrm{e}^{-(M+F)(k+1)}}{1-\mathrm{e}^{-M-F}}-1
\end{gathered}
$$

respectively. Again, $k$ is the maximum age and constants of proportionality have been omitted for these 2 quantities as they are irrelevant to Eq. (6).

Once more, the analytic expression for Eq. (6) derived from Eqs. (15) and (16) simplifies in the limits of $k=1$ and $k=\infty$. In both cases, one finds that reserves will increase yield if the following condition is satisfied:

$$
\frac{1}{F}-\frac{1}{M+F}-\frac{1}{\mathrm{e}^{F}-1}+\frac{1}{\mathrm{e}^{M+F}-1}>0
$$

Numerical evaluation or expansion of the exponentials to second order reveals that this expression is positive for all non-zero values of $M$ and $F$. This indicates that yield will increase with increasing reserve size, no matter how large the reserve. This is somewhat surprising given that the discrete populations examined in the previous subsection showed no such increase despite having quite similar population structures. As will become clearer after the examination of populations with age limits for first reproduction, this increase in yield with reserves is due to the delay between the onset of fishing and first reproduction.

For other values of the maximum age, yield can either increase or decrease depending on the precise values of $M$ and $F$, with high $M$ and/or $F$ favoring increases (Fig. 2). This can be directly demonstrated by considering the MSY of a population with a maximum age of 2 and Beverton-Holt density-dependent recruitment (see Fig. 3 legend for precise parameterization of the density dependence). For small values of $M$, MSY decreases as the area in reserves is increased, but for larger values of $M$, MSY begins to increase with area in reserves (Fig. 3). Comparisons between the values of $F$ that produce MSY at a given $M$ in Fig. 3 and the results in Fig. 1 show that the signs of the changes with fraction of habitat in reserves are correctly predicted by Eq. (6).

Despite this potential for increased total yield, it is important to note that substitution into Eq. (10) reveals that CPUE decreases with increasing reserve size for all values of $M, F$ and $k$.

\section{Allometric growth}

Next, consider a population for whom biomass (i.e. the yield of an individual fish) and reproductive capacity are allometric functions of age. For simplicity, it is assumed that individuals have the potential to live infinitely long and that natural mortality, fishing mortality and reproduction are continuous and simultaneous processes throughout the life of an individual. A necessary corollary of the results for discrete-time populations is that, in the absence of allometric growth, yield is the same with and without reserves for this continuous-time population. This can be seen by considering the limit of infinitely small time steps for the discretetime population with infinite maximum age examined above. Therefore, this population structure provides a

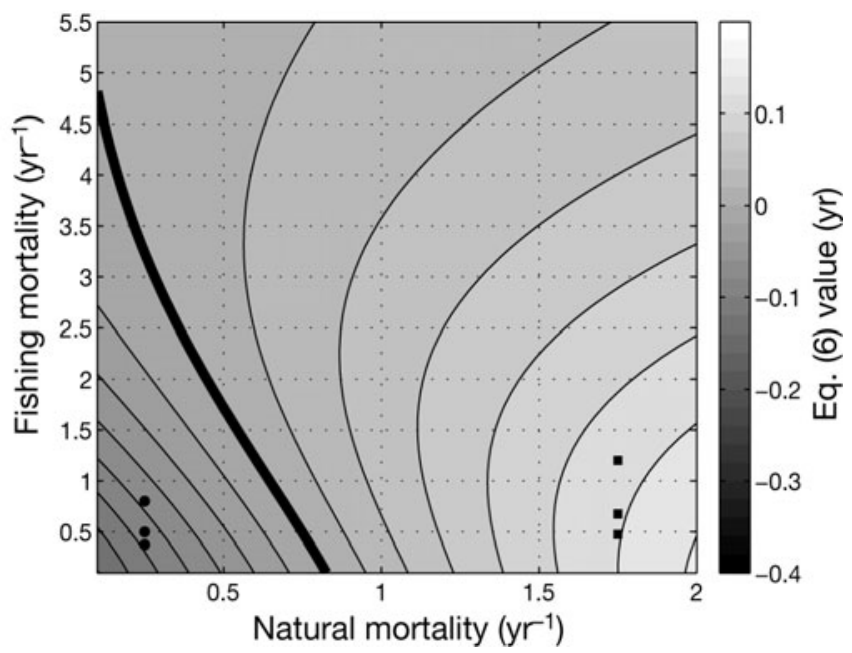

Fig. 2. Value of Eq. (6) for a population with continuous natural and fishing mortality, discrete reproduction and a maximum age of 2 . The thick, black contour indicates the 0 line separating values of natural $(M)$ and fishing $(F)$ mortality that produce increasing yields with marine reserves at fixed total recruitment or maximum substainable yield (MSY) from those that produce decreases in yield. The dots and squares indicate the values of $M$ and $F$ producing MSY in the curves shown in Fig. 3 (a) and (b), respectively 
good system for isolating just the effects of allometric growth on harvests.

The probability of survival of an individual to a certain age is described by:

$$
n(a)=\mathrm{e}^{-(M+F) a}
$$

Biomass and reproductive capacity are given by:

$$
\begin{aligned}
& B(a)=a^{\alpha} \\
& \rho(a)=a^{\beta}
\end{aligned}
$$

where $B(a)$ is the biomass at age $a_{1} \rho(a)$ is the reproductive capacity, and $\alpha$ and $\beta$ are constants. Note that constants of proportionality have not been included in these relationships as these will not contribute to Eq. (6).

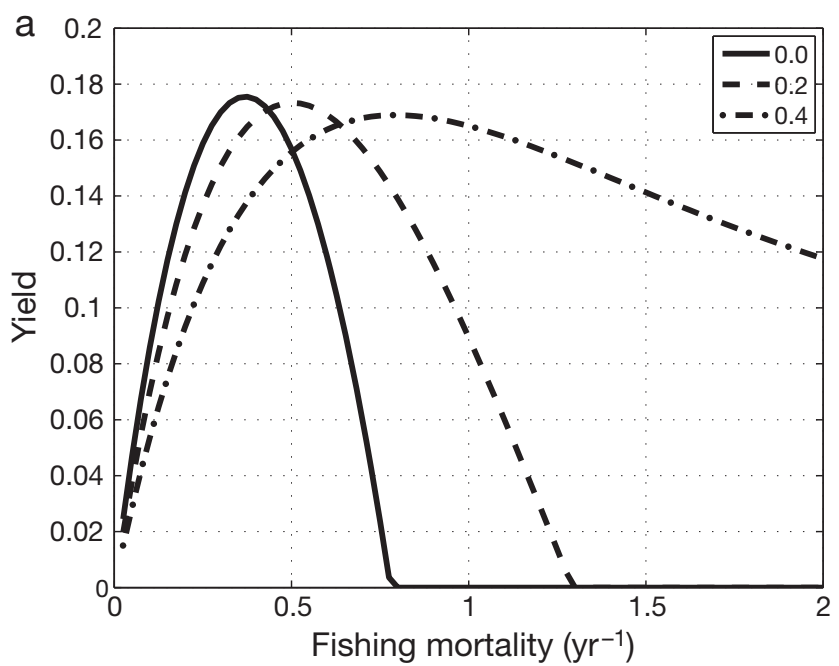

b

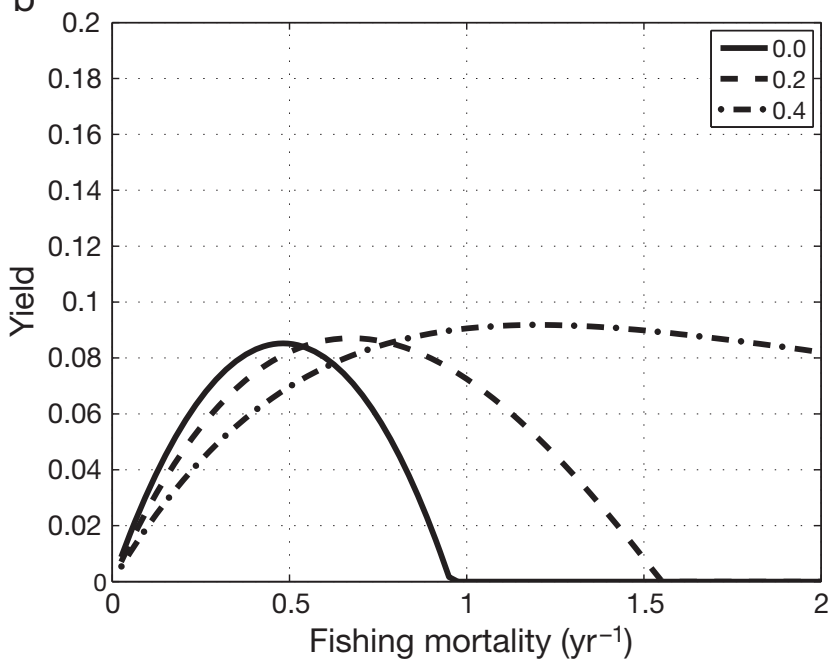

Fig. 3. Yield as a function of fishing mortality for natural mortality rates of (a) $0.25 \mathrm{yr}^{-1}$ and (b) $1.75 \mathrm{yr}^{-1}$ for 3 different fractions of total habitat in reserves: 0.0 (solid), 0.2 (dashed) and 0.4 (dash-dotted). The slope at 0 recruitment and maximum recruitment of the Beverton-Holt density-dependence are 2.1 and 1.0, respectively, for (a) and 14.0 and 1.0, respectively, for (b)
Given these relationships, the YPR and LEP per recruit are:

$$
\begin{gathered}
y_{0}(F)=\int_{0}^{\infty} F \cdot S(a) \cdot n(a) \mathrm{d} a=F \int_{0}^{\infty} \mathrm{e}^{-(M+F) a} \mathrm{a}^{\alpha} \mathrm{d} a= \\
\frac{F}{(M+F)^{\alpha+1}} \int_{0}^{\infty} \mathrm{e}^{-x} X^{\alpha} \mathrm{d} x=\frac{\Gamma(\alpha+1) F}{(M+F)^{\alpha+1}}
\end{gathered}
$$

and

$b_{0}(F)=\int_{0}^{\infty} \rho(a) \cdot n(a) \mathrm{d} a=\int_{0}^{\infty} \mathrm{e}^{-(M+F) a} a^{\beta} \mathrm{d} a=\frac{\Gamma(\beta+1)}{(M+F)^{\beta+1}}$

where $\Gamma(x)$ is the gamma function. As the gamma functions above are independent of the fishing rate, they are irrelevant to Eq. (6) and can be ignored. Substituting these expressions into Eq. (6) yields:

$$
\frac{1}{F}-\frac{\alpha+1}{M+F}-\frac{(\beta+1) M^{\beta+1}}{\left((M+F)^{\beta+1}-M^{\beta+1}\right)(M+F)}
$$

As predicted, this quantity vanishes for $\alpha=\beta=0$ and, therefore, yield is unchanged with reserves for populations with continuous mortality and reproduction in the absence of allometric growth.

For other values of $\alpha$ and $\beta$, yield can either increase or decrease. In general, $\beta>>\alpha$ produces yields with reserves that are higher than those without reserves (Fig. 4). Reserves also tend to produce higher yield when the natural mortality rate is high and/or the fishing rate is low relative to the natural mortality rate (i.e. relatively short-lived species). Nevertheless, Eq. (6) is consistently negative for all values of $M$ and $F$ that were considered for realistic values of $\alpha$ and $\beta$ (dots in Fig. 4, Gunderson 1997). Therefore, in the absence of natural mortality rates well beyond values typically found in nature (e.g. Gunderson 1997) and/or additional life-history factors not included in the model (e.g. age limits discussed in the next section), yields at fixed total recruitment are likely to decrease with marine reserves for most species. This explains in general terms why several modeling studies have found that MSY of typical marine populations decreases with reserves (e.g. Hart 2006, Kaplan 2006, Walters et al. 2007). Furthermore, substitution of Eqs. (20) and (21) into Eq. (10) reveals that the factional change in CPUE is proportional to:

$$
\frac{-\alpha-1}{M+F}
$$

As this quantity is clearly negative, CPUE decreases for all values of $M, F, \alpha$ and $\beta$.

\section{Age limits for reproduction and fishing}

Next, age limits for both reproductive maturity and first harvest are added to the model. While the age at first harvest is not, strictly speaking, a life-history para- 
meter (as it is controlled by the fishery and can in theory be modified by management decisions), in practice it is often the case that it is difficult to change, either due to fishing-gear limitations or because several species are caught in the same fishery (e.g. mixed fisheries or those with by-catch species; see O'Farrell \& Botsford 2006b for one example). As such, it is often useful to consider the advantages of MPAs for particular values of this parameter.
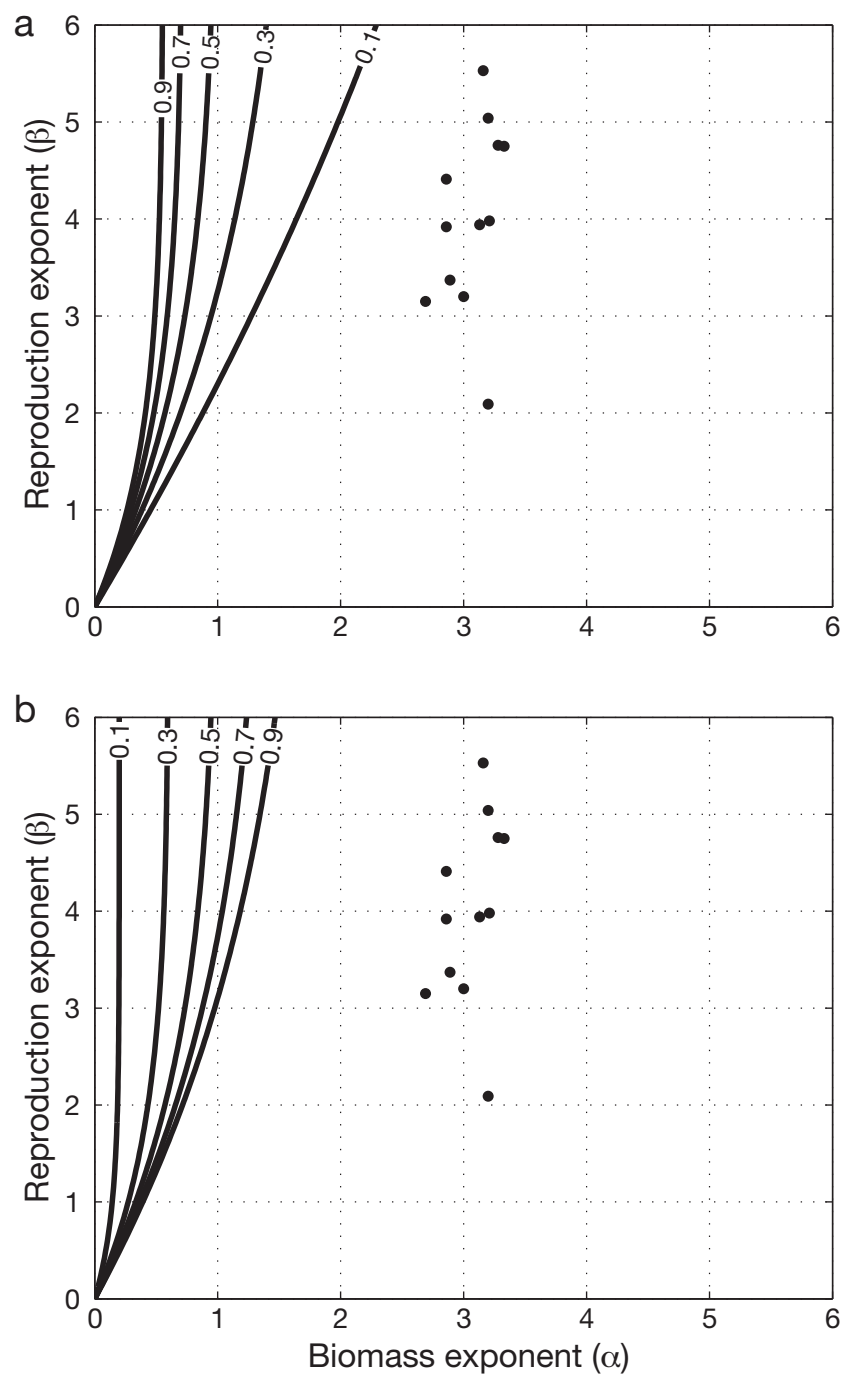

Fig. 4. Contours of when the expression in Eq. (6) $=0$ for a population whose biomass and reproductive capacity are allometric functions of age. The exponent that describes the relationship between biomass and age varies along the $x$-axis, while that for reproductive capacity is along the $y$-axis. (a) Natural mortality is constant at $0.5 \mathrm{yr}^{-1}$ and fishing mortality is varied from 0.1 to $0.9 \mathrm{yr}^{-1}$ (values indicated on contours). (b) Fishing mortality is $0.5 \mathrm{yr}^{-1}$ and natural mortality is varied (values indicated on contours). In both panels, Eq. (6) $>0$ (increased yield) in the upper-left-hand corner of the panel. Dots correspond to values of the biomass and reproductive exponents for several fish species taken from Gunderson (1997) (assuming a linear relationship between age and length)
Considering the life history of a single recruit as a function of age, the probability of survival to a particular age is given by:

$$
\begin{gathered}
n(a)=\mathrm{e}^{-M a} \quad \text { if } a \leq a_{F} \\
n(a)=\mathrm{e}^{-M a} F \mathrm{e}^{-(M+F)\left(a-a_{F}\right)} \quad \text { if } a>a_{F}
\end{gathered}
$$

where $a_{\mathrm{F}}$ is the age of first harvest. The biomass at age relationship is identical to that in the last section. The reproductive capacity as a function of age is given by:

$$
\rho(a)=\left\{\begin{array}{ccc}
0 & \forall & a<a_{50} \\
a^{\beta} & \forall & a \geq a_{50}
\end{array}\right.
$$

where $a_{50}$ is the age at first reproduction (here, for simplicity, I equate this with the age at $50 \%$ fecundity). Note that as reproductive activity before the onset of fishing does not contribute to Eq. (6), one need only consider cases where $a_{50} \geq a_{\mathrm{F}}$.

The YPR, LEP and the value of Eq. (6) for this population were evaluated numerically (details and Matlab scripts available from the author upon request). Results indicate that age limits significantly affect whether or not yield will increase with reserves. If both the age at first capture and the age of first reproduction are increased together, then the range of values of $\alpha$ and $\beta$ that produce higher yields with reserves increases (upper-left part of Fig. 5). This is ultimately due to the fact that for a non-zero age limit, the initial reproductive capacity and biomass of individuals at age of maturity is non-zero and the initial slope of these relationships with age is steeper than with age limits of 0 . Logically it makes sense that the steeper increase in reproductive capacity with age favors protecting older age classes in reserves.

Furthermore, the age of reproductive maturity relative to that of entry into the fishery is important for determining whether or not yield will increase with reserves (Fig. 6). As the difference between the 2 ages increases, a wider range of values of $\alpha$ and $\beta$ have increased yield with reserves, and whether or not reserves increase yield becomes progressively less dependent on the value of $\beta$. In particular, the combination of late entry into the fishery and even later age at first reproduction has the potential to produce higher yields for biologically realistic values of $\alpha$ and $\beta$.

The patterns described above can also be seen by considering the value of Eq. (6) as a function of the ages of reproductive maturity and first harvest for all other parameters fixed (Fig. 7). For realistic values of the mortality rates and $\alpha$ and $\beta$, MPA benefits only occur if the age at first harvest is non-zero and fish mature sexually after entering the fishery. This in turn has consequences for management decisions that change the age at first capture, as the negative impact of reserves on yield for fish that mature sexually before 
entering the fishery suggests that while MPAs may benefit the fishery for late-maturing fish, they may not be globally the optimal choice if gear can be modified to increase the age at first capture.

To gain some insight into these results, it is useful to consider the simple case of $\alpha=\beta=0$. In this case, YPR and LEP are easily evaluated to be:

$$
\begin{gathered}
y_{0}(F) \approx \frac{F}{M+F} \\
b_{0}(F) \approx \frac{\mathrm{e}^{-F\left(a_{50}-a_{F}\right)}}{M+F}
\end{gathered}
$$

where, in both cases, multiplicative factors independent of $F$ have been dropped from the expressions. Substitution into Eq. (6) yields:

$$
\frac{1}{F}-\frac{1+M\left(a_{50}-a_{F}\right) \mathrm{e}^{-F\left(a_{50}-a_{F}\right)}}{F+M\left(1-\mathrm{e}^{-F\left(a_{50}-a_{F}\right)}\right)}
$$

This function is always $\geq 0$ and is an increasing function of $a_{50}-a_{F}$, the difference between the age of entry into the fishery and the age of reproductive maturity.

While these results are specific to the case $\alpha=\beta=0$, it is clear that exponential factors of the form $\mathrm{e}^{-F\left(a_{50}-a_{F}\right)}$ will occur in the expression for LEP for all values of $\alpha$ and $\beta$. These factors generally have a positive effect on Eq. (6) due to the decreasing slope of negative exponentials, explaining the tendency towards increases in yield with reserves for a wider range of $\alpha$ and $\beta$. This also suggests an explanation for

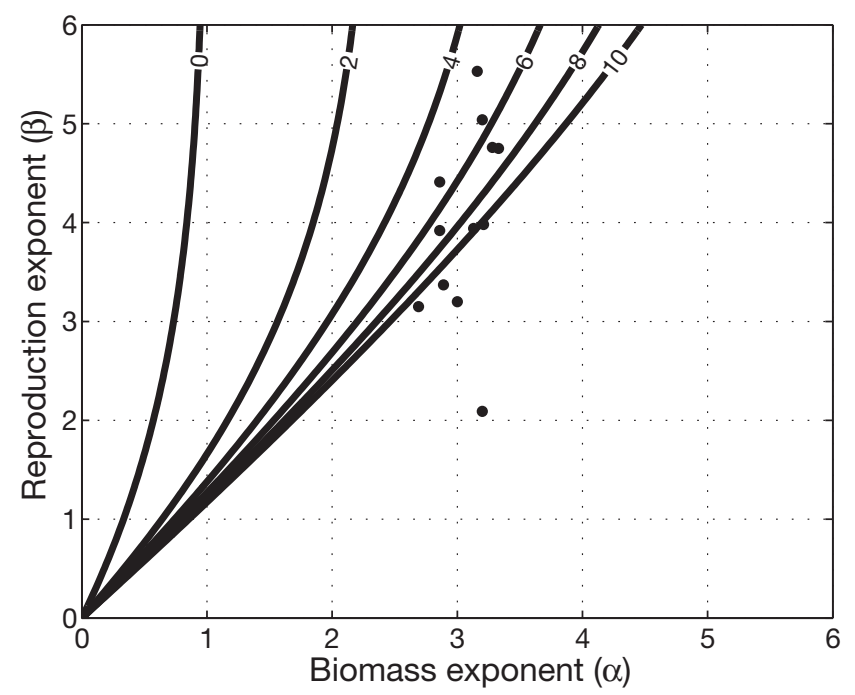

Fig. 5. Zero contours of the expression in Eq. (6) for a population where individuals enter the fishery and become reproductively mature at the same age (age, indicated by value on contour, ranges from 0 to $10 \mathrm{yr}$ ). The natural and fishing mortality rates are both $0.5 \mathrm{yr}^{-1}$. Dots correspond to values of the biomass and reproductive exponents for several fish species taken from Gunderson (1997) (assuming a linear relationship between age and length) the lack of dependence on the value of $\beta$ for large values of $a_{50}-a_{F}$. As in Eq. (27), for large values of $a_{50}-a_{F \prime}$ the contribution of LEP to Eq. (6) becomes solely a function of $M$ and $F . a_{50}-a_{F}$ and $\beta$ evidently do not contribute. This derives from the fact that, for large values of $a_{50}-a_{F}$, only a small fraction of individuals live to reproduce and reproduction occurs over a relatively limited set of ages, thereby reducing the dependence of yield on the functional form of the agevs.-reproductive-capacity relationship.
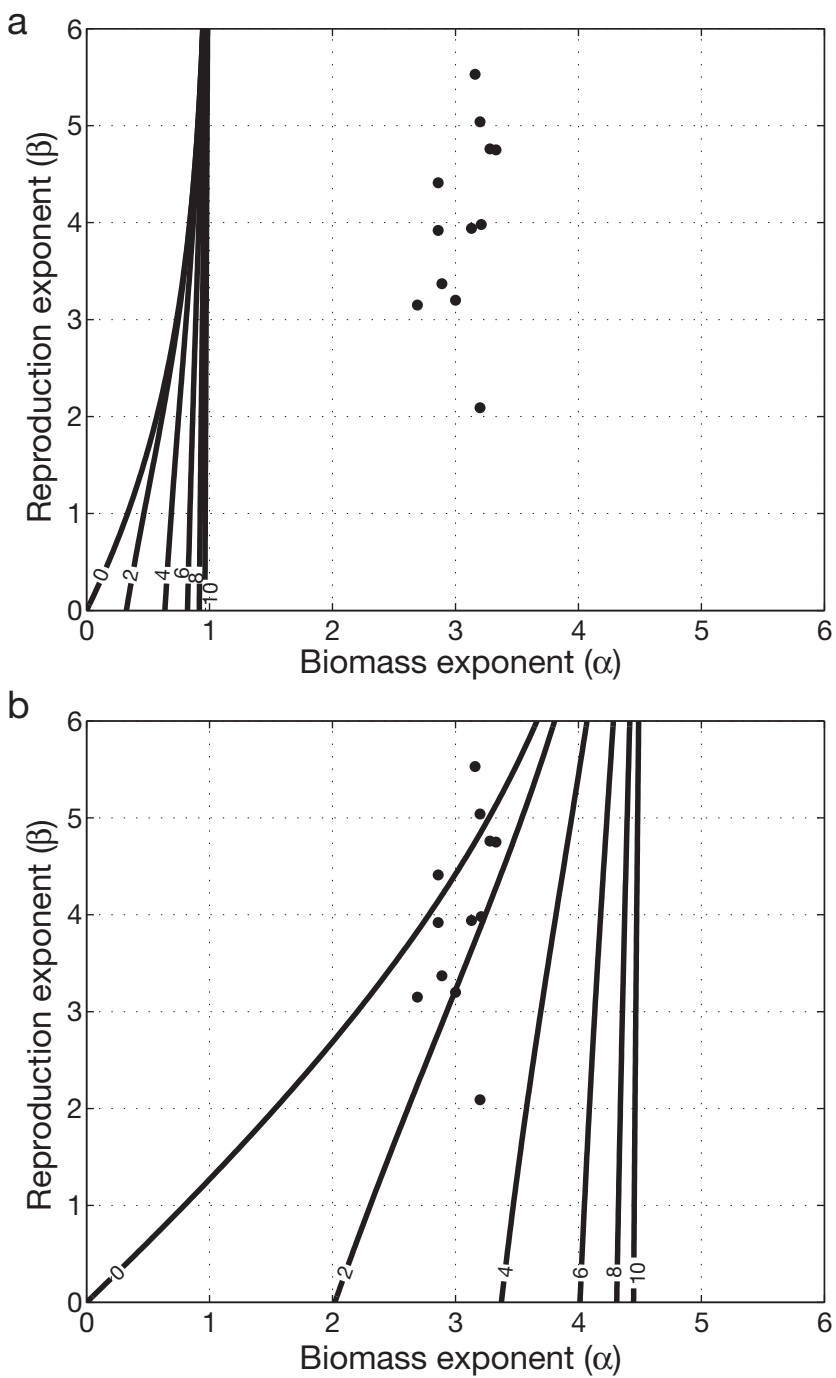

Fig. 6. Zero contours of expression in Eq. (6) for fixed age at first capture for several values of the age of first reproduction. (a) Individuals enter the fishery at age 0. (b) Age at first capture is 6 yr. Values indicated on contours are the age at first reproduction relative to the age of entry into the fishery, i.e. in (b) the contour labeled with a 2 means that the age of first reproduction is actually $8=6+2$. Natural and fishing mortality rates are both $0.5 \mathrm{yr}^{-1}$. Dots correspond to values of the biomass and reproductive exponents for several fish species taken from Gunderson (1997) (assuming a linear relationship between age and length) 


\section{Non-linear Von Bertalanffy growth}

In real fish populations, size as a function of length generally plateaus at a certain maximum size. This is often represented by a non-linear von Bertalanffy growth curve:

$$
L(a)=L_{\infty}\left(1-\mathrm{e}^{-\kappa a}\right)
$$

where $L_{\infty}$ is the maximum size and $\kappa$ is the growth rate. The maximum size will drop out of Eq. (6), and, therefore, will not be mentioned further. Allometric relationships are used to describe biomass and reproductive capacity as a function of length:

$$
B(a)=L^{\alpha}
$$

and

$$
\rho(a)=\left\{\begin{array}{lll}
0 & \forall & a<a_{50} \\
L^{\beta} & \forall & a \geq a_{50}
\end{array}\right.
$$

This population reduces to that of the previous section in the limit that $\kappa<<+F$, as most individuals do not reach the maximum size during their lifetimes and the von Bertalanffy growth curve will be approximately linear over the range of ages most individuals experience. Therefore, yield trends are similar to those in the previous section for small values of $\kappa$. Furthermore, this population reduces to that discussed at the end of the previous section, with $\alpha=\beta=0$ in the limit that $\kappa>>M+$ $F$ as individuals are at the maximum size during most of their lifetimes. As yield increases with reserves when $\alpha=\beta=0$ (Eq. [27]), it is not surprising that numerical

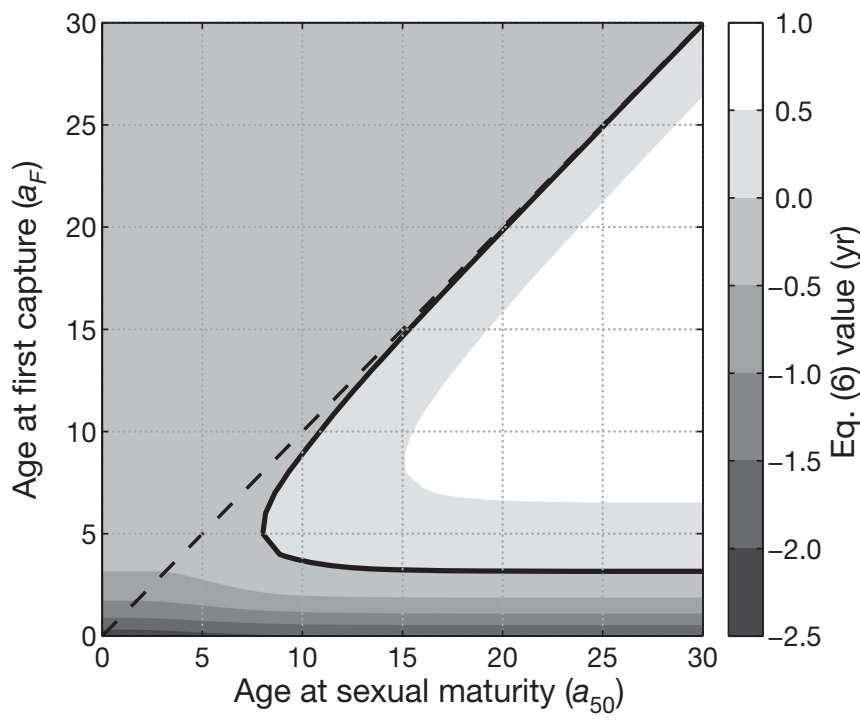

Fig. 7. Value of Eq. (6) as a function of age at sexual maturity $\left(a_{50}\right)$ and age at first harvest $\left(a_{F}\right)$ for fixed natural and fishing mortality rates (both $0.5 \mathrm{yr}^{-1}$ ), and biomass and reproductive exponents (both 3). The solid thick curve indicates the zero contour, while the dashed line indicates for reference the line with equal ages at sexual maturity and first capture evaluation of Eq. (6) shows that the range of $\alpha$ and $\beta$ producing increased yields with reserves expands as $\kappa$ is increased (Fig. 8). However, trade-offs between reproduction and survival tend to limit the possible parameter values and it is unlikely that $\kappa$ is much larger than $M$ in real species (Jensen 1996, 1997).

Despite these potential increases in total yield with reserves for fast growing species, CPUE decreased universally with reserves for all parameter values examined with and without von Bertalanffy growth.

\section{DISCUSSION}

Here it has been shown that whether reserves combined with effective conventional management (here defined as management for fixed overall recruitment or MSY) will increase total yield for a population with sedentary adults, widely dispersing larvae and intracohort density-dependent recruitment is governed by a simple equation that compares the sensitivities of YPR and LEP to changes in the fishing rate. This result has relevance for a wide variety of species, provides a natural complement to the results of Hart (2006) for when MPAs increase yield at fixed fishing rate, and addresses the vital question of how MPAs will affect well-managed species. This simple approach is not intended to produce detailed predictions of MPA effects. Rather, this result is viewed as providing a vital indicator for identifying species with life-history traits that favor the use of MPAs. As life-history traits are likely to have similar overall effects no matter what specific conventional management strategy is used, the approach has value for management scenarios beyond those directly considered here.

The potential of this approach for providing a simple benchmark of MPA effects on fishery harvests rests in its applicability to a wide variety of species. As Eqs. (6) and (10) are independent of the settler-recruit relationship, which is generally poorly understood and subject to large uncertainties, they can be estimated even in data-limited situations. The basic life-history data required to estimate the sensitivities are relatively easy to obtain. Age-length relationships and biomass/reproduction as a function of length can often be evaluated in the laboratory. Estimates of mortality rates are available in many cases, though with non-negligible uncertainties. The basic approach followed here of evaluating Eq. (6) for a wide variety of parameter values to assess the sensitivity of results to different parameterizations may be of value in addressing these uncertainties.

The results presented here link previous studies indicating that yield would be equivalent with and without reserves (Hastings \& Botsford 1999) with results from more complex models showing that yield 


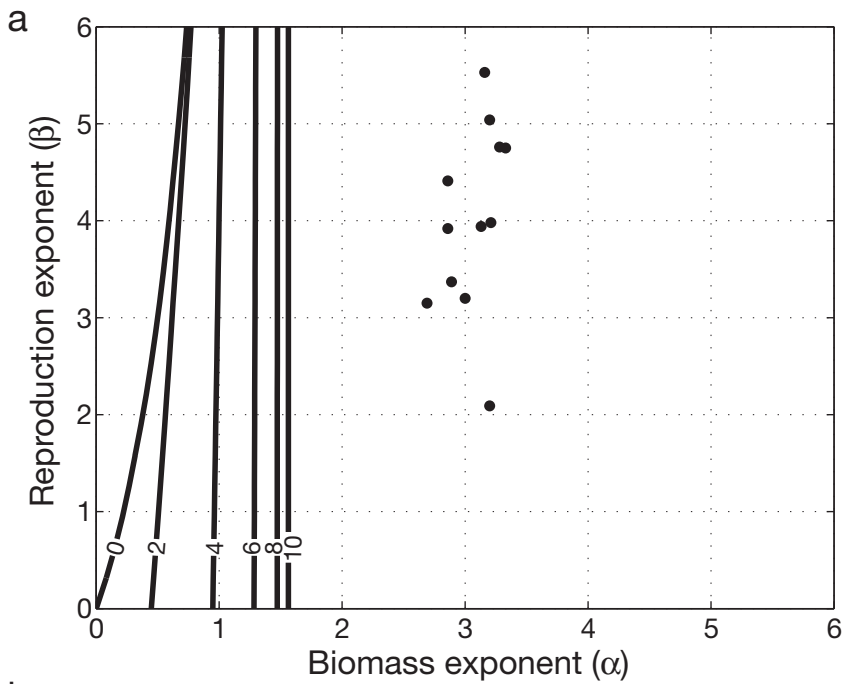

b

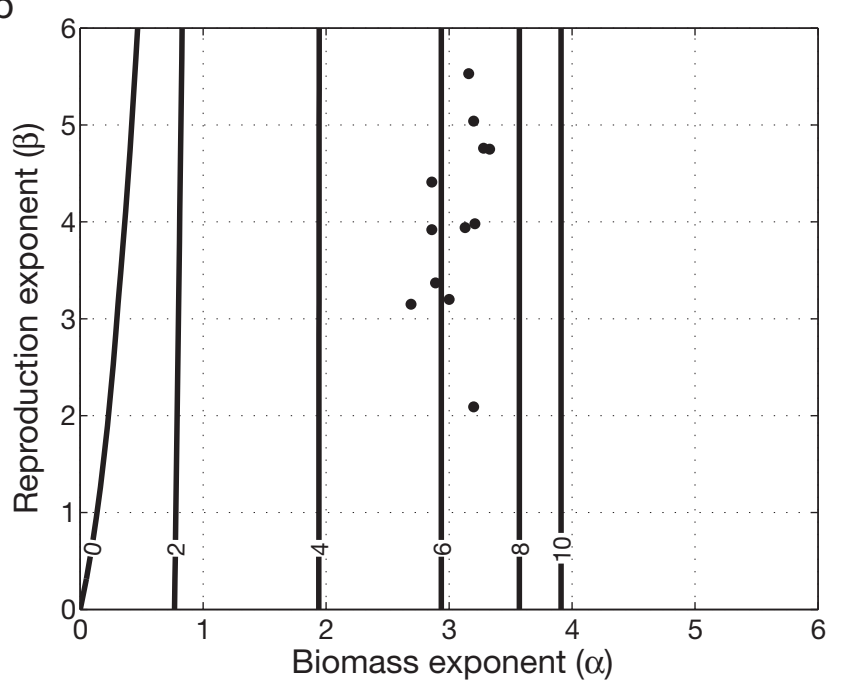

Fig. 8. Zero contours of expression in Eq. (6) for population with von Bertalanffy growth. (a) Value of the growth rate $\kappa=$ 0.5. (b) $\kappa=1.5$. Age at first capture is $0 \mathrm{yr}$. Values indicated on contours are the age at first reproduction. The natural and fishing mortality rates are both $0.5 \mathrm{yr}^{-1}$. Dots correspond to values of the biomass and reproductive exponents for several fish species taken from Gunderson (1997)

of well-managed fisheries is likely to decline with reserves (e.g. Hart 2006, Kaplan 2006, Walters et al. 2007). Though yield is the same with and without reserves for simple populations that are semelparous or have age-independent mortality and size, yield for populations with age-dependent growth and mortality (including finite life expectancy) is a complex function of life-history parameters. Both positive and negative results are possible, but decreases in yield are more probable for realistic age-weight relationships, explaining many of the negative MPA results in previous modeling studies.

One factor common to those combinations of population structure and life-history parameters that did produce higher yield of effectively managed species with MPAs was a 'mismatch' between the various vital parameters. Examples include reproductive growth rates much higher than biomass growth rates (i.e. $\beta$ >> $\alpha$ ), first harvest occurring well before first reproduction, and the von Bertalanffy growth rate much higher than the mortality rates (i.e. $\kappa>>M+F$ ). While in theory these combinations are possible, in practice bio-energetic considerations and trade-offs between reproduction and survival bound possible values and limit these mismatches (Jensen 1996, 1997). Only relatively few realistic combinations of population structure and life-history parameters have the potential to produce harvests increases at fixed recruitment or MSY. Late age of entry into the fishery and even later age of first reproduction both favor marine reserves, particularly when combined with rapid growth to maximum size (relative to life expectancy and independent of the age at reproductive maturity). There are a number of fish and fisheries that may satisfy these conditions. California rockfish Sebastes spp. are typically long-lived and only reach reproductive maturity after several years (Love et al. 2002). Furthermore, fisheries harvest of reproductively immature individuals of several rockfish species is significant (e.g. O'Farrell \& Botsford 2006a). Similarly, the Gulf of Lions (Mediterranean) trawling fishery for hake is almost entirely based on catch of juveniles (French-Spanish Working Group 2006). If harvest of individuals before reproductive maturity cannot be controlled by appropriate fishing gear changes, then the results presented here strongly suggest that reserves may provide an effective fishery management strategy for these species.

One aspect of fish life histories often cited as potentially favoring management with MPAs is the increased reproductive capacity of Big Old Fecund Females (BOFFs; Berkeley et al. 2004). It has been shown for several fish species that older females have higher than expected egg production, egg size and larval survival rates (e.g. Hislop 1988, Trippel 1998, Berkeley et al. 2004). Overall, these effects lead to a (previously unrecognized) increase in reproductive capacity for older individuals. This increase can be approximated by a higher than originally estimated value of $\beta$, the exponent describing the functional dependence of reproductive capacity on size. Larger values of $\beta$ do in fact favor management with reserves. However, in the absence of other effects, such as late age of reproductive maturity, extremely large values of $\beta$ are required to increase total yield with MPAs. This suggests that the BOFF effect alone (in the absence of additional factors not considered in this model, such as inter-cohort density-dependence) is not sufficient to produce higher yields with reserves in well-managed fisheries, consistent with the results of O'Farrell \& Botsford (2006b). 
It has recently been recognized that inter-cohort density-dependent larval recruitment (i.e. recruitment depending on local adult density) can produce greater harvests with reserves than via conventional management alone (Gaylord et al. 2005, White \& Kendall 2007, White et al. 2008). This is because harvest of adults removes competitive pressure on new recruits; therefore, increases in the fishing rate can increase recruitment if reserves are sufficient to ensure larval production. The strength of such inter-cohort density dependence is difficult to quantify for most species and there are undoubtedly species for which intracohort density-dependence, as used here, is dominant (see the review in Caley et al. 1996 for evidence for and against the importance of inter-cohort density dependence). Nevertheless, this alternative mechanism favoring the use of MPAs may be important for marine species (e.g. as suggested by Hobson et al. 2001). Unfortunately, inter-cohort density dependence cannot easily be incorporated into the approach used here. Nevertheless, as this mechanism is based on changes in recruitment, whereas those identified here (e.g. late reproductive maturity) are mainly related to the adult part of the life cycle, I suspect that these mechanisms are complimentary and species that exhibit both may do particularly well when managed with MPAs (e.g. potentially, California rockfish; Hobson et al. 2001).

Despite increased yields with reserves for certain lifehistory traits, CPUE went down in the model for all parameter values and population structures considered. As pointed out by numerous resource economists, increases in total yield are not sufficient to assure that fishers will benefit from MPAs (e.g. Sanchirico et al. 2006). If CPUE goes down, then the profitability of fishing will likely decrease. The results in this paper indicate that while global increases in yield are possible for some life histories, MPAs are unlikely to be optimal in terms of profits in the absence of other factors, such as overfishing (Smith \& Wilen 2003) or inter-cohort density dependence (White et al. 2008).

The assumptions made in the model used here of well-mixed larvae and no adult movement are approximately valid for a variety of species (Larson et al. 1994, Jorgensen et al. 2006), but there is evidence that local larval retention (Almany et al. 2007) and adult movement (McClanahan \& Mangi 2000) are non-negligible in many species. Though not explicitly treated in this study, the consequences of modifying these assumptions can, however, be anticipated. In the limit of no larval dispersal, larvae produced inside reserves stay in reserves and, therefore, do not contribute to fished populations outside. Reserves represent a loss to the fishery in this case. Therefore, limited larval dispersal will likely produce higher yields via conventional man- agement alone than with MPAs (though the conservation benefits of reserves may increase if dispersal is limited). For species with very limited adult movement compared to the reserve size, results will not be terribly different than those found here. For species with more extensive adult movement, reserves will not provide significant protection as all individuals will be accessible to the fishery. Therefore, one expects little change in total yield with reserves for species that move considerably as adults.

Acknowledgements. This work was in part supported by a grant from the Resource Legacy Funding Foundation. The author thanks L. W. Botsford and M. R. O'Farrell for their comments that helped initiate this research, as well as D. Hart and the anonymous reviewers for their many insightful recommendations.

\section{LITERATURE CITED}

Adams PB (1980) Life-history patterns in marine fishes and their consequences for fisheries management. Fish Bull (Wash DC) 78:1-12

Almany GR, Berumen ML, Thorrold SR, Planes S, Jones GP (2007) Local replenishment of coral reef fish populations in a marine reserve. Science 316:742-744

Berkeley SA, Chapman C, Sogard SM (2004) Maternal age as a determinant of larval growth and survival in a marine fish, Sebastes melanops. Ecology 85:1258-1264

Caley MJ, Carr MH, Hixon MA, Hughes TP, Jones GP, Menge BA (1996) Recruitment and the local dynamics of open marine populations. Annu Rev Ecol Syst 27:477-500

French-Spanish Working Group (2006) Assessment of hake (Merluccius merluccius) exploited by the French and Spanish fisheries developed in GSA 07 (Gulf of Lions), 11-14 September 2006, In: Mellon C, Jadaud A, Farrugio H, Massutí E and others (eds) Report of the eight sessions of the Sub-Committee on Stock Assessment (SCSA). FAO, Rome. Available at ftp://ftp.fao.org/fi/DOCUMENT/gfcm/sac9/inf8e.pdf

Gaylord B, Gaines SD, Siegel DA, Carr MH (2005) Marine reserves exploit population structure and life history in potentially improving fisheries yields. Ecol Appl 15:2180-2191

Gerber LR, Botsford LW, Hastings A, Possingham HP, Gaines SD, Palumbi SR, Andelman S (2003) Population models for marine reserve design: a retrospective and prospective synthesis. Ecol Appl 13:S47-S64

> Gerber LR, Heppell SS, Ballantyne F, Sala E (2005) The role of dispersal and demography in determining the efficacy of marine reserves. Can J Fish Aquat Sci 62:863-871

Gunderson DR (1997) Trade-off between reproductive effort and adult survival in oviparous and viviparous fishes. Can J Fish Aquat Sci 54:990-998

Hart DR (2006) When do marine reserves increase fishery yield? Can J Fish Aquat Sci 63:1445-1449

Hastings A, Botsford LW (1999) Equivalence in yield from marine reserves and traditional fisheries management. Science 284:1537-1538

- Hislop JRG (1988) The influence of maternal length and age on the size and weight of the eggs and the relative fecundity of the haddock, Melanogrammus aeglefinus, in British waters. J Fish Biol 32:923-930

Hobson ES, Chess JR, Howard DF (2001) Interannual variation in predation on first-year Sebastes spp. by three northern California predators. Fish Bull (Wash DC) 99:292-302 
Jennings S, Reynolds JD, Mills SC (1998) Life history correlates of responses to fisheries exploitation. Proc R Soc Lond B Biol Sci 265:333-339

Jensen A (1996) Beverton and Holt life history invariants result from optimal trade-off of reproduction and survival. Can J Fish Aquat Sci 53:820-822

> Jensen A (1997) Origin of the relation between K and L-inf and synthesis of relations among life history parameters. Can J Fish Aquat Sci 54:987-989

Jorgensen SJ, Kaplan DM, Klimley AP, Morgan SG, O'Farrell MR, Botsford LW (2006) Limited movement in blue rockfish Sebastes mystinus: internal structure of home range. Mar Ecol Prog Ser 327:157-170

Kaplan DM (2006) Alongshore advection and marine reserves: consequences for modeling and management. Mar Ecol Prog Ser 309:11-24

Kaplan DM, Botsford LW, Jorgensen S (2006) Dispersal-perrecruit: an efficient method for assessing sustainability in marine reserve networks. Ecol Appl 16:2248-2263

Larson RJ, Lenarz WH, Ralston S (1994) The distribution of pelagic juvenile rockfish of the genus Sebastes in the upwelling region off Central California. CCOFI Rep 35:175-221

Love MS, Yoklavich M, Thorsteinson L (2002) The Rockfishes of the Northeast Pacific. University of California Press, Berkeley, CA

Mace PM (2001) A new role for MSY in single-species and ecosystem approaches to fisheries stock assessment and management. Fish Fish 2:2-32

McClanahan TR, Mangi S (2000) Spillover of exploitable fishes from a marine park and its effect on the adjacent fishery. Ecol Appl 10:1792-1805

O'Farrell MR, Botsford LW (2006a) Estimating the status of nearshore rockfish (Sebastes spp.) populations with length frequency data. Ecol Appl 16:977-986

O'Farrell MR, Botsford LW (2006b) The fisheries management implications of maternal-age-dependent larval survival. Can J Fish Aquat Sci 63:2249-2258

Sale PF, Cowen RK, Danilowicz BS, Jones GP and others (2005) Critical science gaps impede use of no-take fishery reserves. Trends Ecol Evol 20:74-80

Sanchirico JN, Malvadkar U, Hastings A, Wilen JE (2006) When are no-take zones an economically optimal fishery management strategy? Ecol Appl 16:1643-1659

Smith MD, Wilen J (2003) Economic impacts of marine reserves: the importance of spatial behavior. J Environ Econ Manag 46:183-206

Trippel EA (1998) Egg size and viability and seasonal offspring production of young Atlantic cod. Trans Am Fish Soc 127:339-359

Walters CJ, Hilborn R, Parrish R (2007) An equilibrium model for predicting the efficacy of marine protected areas in coastal environments. Can J Fish Aquat Sci 64:1009-1018

White C, Kendall BE (2007) A reassessment of equivalence in yield from marine reserves and traditional fisheries managament. Oikos 116:2039-2043

White C, Kendall BE, Gaines S, Siegel DA, Costello C (2008) Marine reserve effects on fishery profit. Ecol Lett 11: $370-379$

Appendix 1. Condition for increased maximum sustainable yield (MSY) with reserves

Hart (2006) determined the partial derivatives of yield with respect to fishing rate and fraction of habitat in reserves for the model used in this paper via implicit differentiation:

$$
\begin{gathered}
\frac{\partial Y}{\partial F}=(1-C) R\left[y_{0}^{\prime}(F)+y_{0}(F) \frac{s^{\prime}(S)(1-C) b_{0}^{\prime}(F)}{1-s^{\prime}(S) b}\right] \\
\frac{\partial Y}{\partial C}=y_{0}(F) R\left[\frac{S^{\prime}(S)(1-C)\left[b_{0}(0)-b_{0}(F)\right]}{1-s^{\prime}(S) b}-1\right]
\end{gathered}
$$

where $R$ and $Y$ are the average recruitment and yield, respectively, per unit of habitat area at equilibrium, $s(S)$ is the post-settlement density dependence function at settlement rate $S, F$ is the fishing mortality rate, $C$ is the fraction of the habitat area in reserves, and $y_{0}(F)$ and $b_{0}(F)$ are the yield per recruit (YPR) and lifetime egg production (LEP) per recruit, respectively, as a function of the local fishing rate.

Maximum sustainable yield (MSY) at fixed area in reserves is defined by the condition that:

$$
\left.\frac{\partial Y}{\partial F}\right|_{C}=0
$$

This condition implicitly defines Fin terms of $C$ and other lifehistory parameters. From the first line of Eq. (A1) (Eq. 11 of Hart 2006), this yields the following condition for MSY:

$$
\frac{s^{\prime}(S)(1-C)}{1-s^{\prime}(S) b}=\frac{-y_{0}^{\prime}(F)}{b_{0}^{\prime}(F) y_{0}(F)}
$$

The question we wish to address is when the total derivative of $Y$ with respect to $C$ is positive given the condition in Eq. (A2):

$$
\begin{gathered}
\left.\frac{\mathrm{d} Y}{\mathrm{~d} C}\right|_{M S Y}=\frac{\mathrm{d} Y}{\mathrm{~d} C} \frac{\mathrm{d} C}{\mathrm{~d} C}+\frac{\mathrm{d} Y}{\mathrm{~d} F} \frac{\mathrm{d} F}{\mathrm{~d} C} \\
\left.\frac{\mathrm{d} Y}{\mathrm{~d} C}\right|_{M S Y}=\frac{\mathrm{d} Y}{\mathrm{~d} C}
\end{gathered}
$$

Therefore, MSY will increase with area in reserves if the partial derivative of yield with respect to fraction of habitat in reserves is positive. Using the second line of Eq. (A1) (Eq. 12 of Hart 2006) for the partial derivative of yield with respect to fraction of the coastline in reserves produces:

$$
\left.\frac{\mathrm{d} Y}{\mathrm{~d} C}\right|_{M S Y}=-y_{0}(F) R\left[\frac{y_{0}^{\prime}(F)}{b_{0}^{\prime}(F)} \frac{\left[b_{0}(0)-b_{0}(F)\right]}{y_{0}(F)}+1\right]
$$

As YPR and the recruitment level are necessarily positive, this is $>0$ when:

$$
0<\frac{y_{0}^{\prime}(F)}{-b_{0}^{\prime}(F)} \frac{\left[b_{0}(0)-b_{0}(F)\right]}{y_{0}(F)}-1=\frac{\frac{\mathrm{d}\left[\ln \left(y_{0}(F)\right)\right]}{\mathrm{d} F}}{\frac{\mathrm{d}\left[\ln \left(b_{0}(0)-b_{0}(F)\right)\right]}{\mathrm{d} F}}-1
$$

As $b_{0}(F)$ is assumed to be a decreasing function of $F$, this expression can be reduced to:

$$
0<\frac{\mathrm{d}\left[\ln \left(y_{0}(F)\right)\right]}{\mathrm{d} F}-\frac{\mathrm{d}\left[\ln \left(b_{0}(0)-b_{0}(F)\right)\right]}{\mathrm{d} F}
$$

This expression is identical to that for increased yield with management for fixed total recruitment. Therefore, the same expression applies to both types of management considered in this study (MSY and fixed recruitment level). Furthermore, as the total derivative of yield with respect to area in reserves reduces to the partial derivative when at MSY (Eq. A4), this condition is an equivalent to that developed in Hart (2006) (specifically Eq. 15) but the condition developed here is only valid at MSY. 